\title{
SeCUElas de Histoplasmosis PUlMONAR
}

\author{
James M. Yurgaky MD. Internista ${ }^{* *}$, Ana María Arredondo G. MD.² \\ ${ }^{1}$ Residente del programa de Endocrinología, Facultad de Medicina, Universidad Militar Nueva Granada, Bogotá., Colombia. \\ ${ }^{2}$ Residente del programa de Medicina Interna, Facultad de Medicina, Universidad Militar Nueva Granada, Bogotá, Colombia
}

\begin{abstract}
Resumen
Radiografía de Tórax en la que se ven múltiples lesiones calcificadas distribuidas en ambos campos pulmonares con la presentación clásica en "perdigones" que corresponden a granulomas en fase de calcificación, esta apariencia radiológica es considerada clásica y patognomónica de histoplasmosis pulmonar; el único diagnóstico diferencial radiológico es la neumonía por varicela la cual es invariablemente sintomática.
\end{abstract}

Palabras clave: histoplasmosis, granuloma, enfermedades pulmonares fúngicas

\section{SEQUELAE OF PULMONARY HISTOPLASMOSIS}

\begin{abstract}
A chest $X$ ray in where they are multiple calcified lesions distributed in both lungs which the classic "pellets" presentation that are calcified granulomas, this radiologic presentation is considered classic and pathognomonic of pulmonary histoplasmosis, the only radiologic differential diagnosis is chickenpox pneumonia which is always symptomatic.
\end{abstract}

Key words: histoplasmosis, granuloma, lung disease, fungal

\section{SEQÜELAS DE HISTOPLASMOSE PULMONAR}

\begin{abstract}
Resumo
Radiografia de tórax em que múltiplas lesões calcificadas são distribuídos em ambos pulmões com a apresentação clássica em "perdigones" que correspondem a granulomas no processo de calcificação, o aspecto radiológico é considerado clássica e patognomônico de histoplasmose pulmonar, o único diagnóstico diferencial radiológico é a pneumonia pela varicela, que é invariavelmente sintomático.
\end{abstract}

Palavras-chave: histoplasmose, granuloma, pneumopatias fúngicas

La histoplasmosis fue descrita por Samuel Darling en Panamá alrededor de 1906; es causada por el hongo dimórfico Histoplasma capsulatum variedad capsulatum, el cual habita los suelos contaminados con excremento de aves y murciélagos. Su presentación clínica es de carácter variable y el estado inmunológico del paciente así como el tamaño del inoculo son los dos factores que determinan el curso de la enfermedad después de la infección primaria. Aproximadamente el $90 \%$ de los pacientes infectados son asintomáticos y el diagnóstico se establece en forma incidental a través de imágenes diagnósticas $(1,2)$.

Correspondencia: JJames Yurgaky jamesy7656@yahoo.es. Dirección correspondencia: Tr. 3 No 49-00, Servicio de Endocrinología, Hospital Militar

Central, Bogotá, Colombia. Teléfono: 4344842 


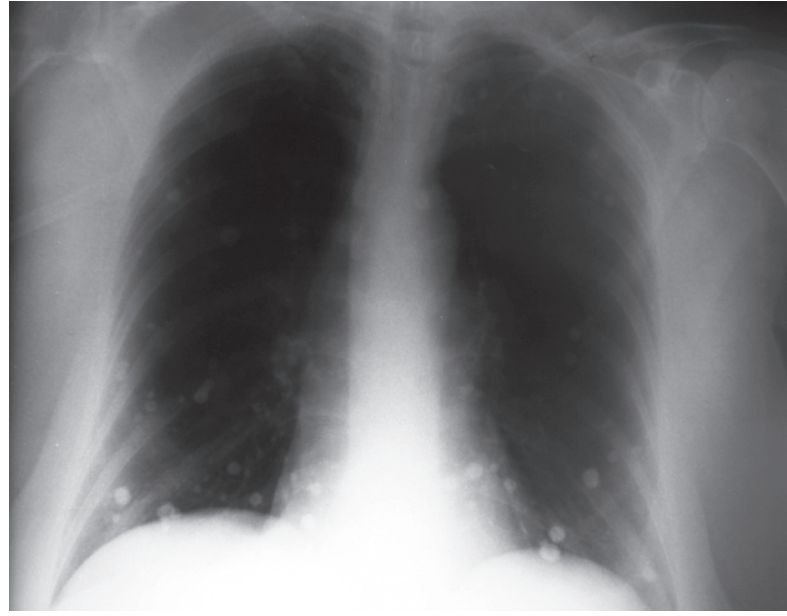

Figura 1. Histoplasmosis pulmonar.

Los granulomas calcificados en histoplasmosis pulmonar adquieren una apariencia descrita como en "perdigones" $(3,4)$. esta En la figura 1 se presenta una radiografía de tórax en proyección anteroposterior que documenta la presencia de lesiones calcificadas, diseminadas en ambos campos pulmonares, las cuales son consideradas patognomónicas de esta enfermedad. El único diagnóstico alternativo para esta presentación radiológica es la neumonía por varicela la cual es invariablemente sintomática, a diferencia de los pacientes con secuelas de histoplasmosis pulmonar y esta presentación radiológica que no tienen un antecedente de neumonía (5-10).

\section{Referencias}

1. Jiménez R, Uran M, de Bedout C, Arango M, Tobón A, Cano Luz, et al. Brote de histoplasmosis aguda en un grupo familiar: identificación de la fuente de infección. Biomédica. 2002; 22:155.

2. Goodwin, R. A., Jr., J. E. Loyd, and R. M. des Prez. 1981. Histoplasmosis in normal hosts. Medicine (Baltimore) 60:231.

3. Goodwin, R. A., Jr., J. L. Shapiro, G. H. Thurman, S. S. Thurman, and R. M. des Prez. 1980. Disseminated histoplasmosis: clinical and pathological correlations. Medicine (Baltimore) 59:1.

4. Gurney, J. W., and D. J. Conces, Jr. 1996. Pulmonary histoplasmosis. Radiology 199:297.

5. Gutierrez, M. E., A. Canton, N. Sosa, E. Puga, and L. Talavera. 2005. Disseminated histoplasmosis in patients with AIDS in Panama: a review of 104 cases. Clin. Infect. Dis. 40:1199.

6. Cano, M., and R. A. Hajjeh. 2001. The epidemiology of histoplasmosis: a review. Semin. Respir. Infect. 16:109

7. Goodwin, R. A., Jr., F. T. Owens, J. D. Snell, W. W. Hubbard, R. D. Buchanan, R. T. Terry, and R. M. des Prez. 1976. Chronic pulmonary histoplasmosis. Medicine (Baltimore) 55:413..

8. Kataria, Y. P., P. B. Campbell, and B. T. Burlingham. 1981. Acute pulmonary histoplasmosis presenting as adult respiratory distress syndrome: Effect of therapy on clinical and laboratory features. South. Med. J. 74:534.

9. Wheat, L. J., and C. A. Kauffman. 2003 Histoplasmosis. Infect. Dis. Clin. N. Am. 17:1.

10. Wheat, L. J. 2001. Laboratory diagnosis of histoplasmosis: update 2000. Semin. Respir. Infect. 16:131. 